\title{
A brief study of Nox 4 inhibitors in diabetic nephropathy
}

\author{
Navneet O. Soni* \\ Department of Pharmacology, NPDCH, Visnagar, Gujarat, India \\ Received: 30 July 2021 \\ Revised: 15 September 2021 \\ Accepted: 22 September 2021 \\ *Correspondence: \\ Dr. Navneet O. Soni, \\ Email: Navneetsoni1978@yahoo.com \\ Copyright: (c) the author(s), publisher and licensee Medip Academy. This is an open-access article distributed under \\ the terms of the Creative Commons Attribution Non-Commercial License, which permits unrestricted non-commercial \\ use, distribution, and reproduction in any medium, provided the original work is properly cited.
}

\begin{abstract}
The purpose of the study was to find the merits and demerits of NADPH oxidase 4 (Nox 4) inhibitors. Nox inhibitors are tested from natural sources like green tea, plumbagin, Chinese formulas like Baoshenfang, Cudrania tricuspidata, Huangqi decoction and synthetic dual Nox inhibitors. Some of them activate and regulate AMP-activated protein kinase (AMPK). Some chunk the high glucose activated alleyway, dawdling the succession of diabetic nephropathy (DN). Overall, the benefits of NOX are: reducing oxidative damage, improving renal function, reducing podocyte injury, preventing interstitial fibrosis, regulating AMPK which inhibits reactive oxygen species (ROS) and transforming growth factor-beta (TGF-beta), decreasing inflammation due to high glucose, protecting mesangial cells, decreasing collagen synthesis, and reducing glomerular hypertrophy. While targeting NOX in renal impairment, off-target effects, especially cardiovascular effects, are one of the major hurdles since diabetes mellitus (DM) is associated with co-morbid cardiovascular problems.
\end{abstract}

Keywords: Diabetic nephropathy, NADPH oxidase, NOX 4, Oxidative stress

\section{INTRODUCTION}

\section{Diabetes mellitus}

Diabetes is a chronic metabolic disease characterized by high levels of blood glucose (or blood sugar), which causes serious damage to the heart, blood vessels, eyes, kidneys, and nerves over time. ${ }^{1-7}$ Type 2 diabetes is the most common, usually affects adults, and occurs when the body develops insulin resistance or fails to produce adequate insulin. ${ }^{1-12}$ The occurrence of type 2 diabetes has increased spectacularly in countries of all income levels over the last three decades. ${ }^{14}$ Type 1 diabetes, also cognized as juvenile diabetes or insulin-dependent diabetes, is a inveterate disease in which the pancreas fabricates very little or no insulin on its own. ${ }^{15-17}$ Access to reasonably priced treatment, including insulin, is critical for people living with diabetes. ${ }^{18-20}$ By 2025, there will be a global agreement to halt the rise in diabetes and obesity. ${ }^{21}$
Diabetes affects roughly 422 million individuals worldwide, with the preponderance living in low-and middle-income nations, and diabetes is directly accountable for 1.6 million deaths each year. ${ }^{22}$ Diabetes has been gradually escalating in both the number of cases and occurrence over the last few decades. ${ }^{22}$

\section{Diabetic nephropathy (DN)}

Diabetic nephropathy is a chronic condition that develops over time and is characterised by gradually increasing urinary albumin excretion (UAE), blood pressure and cardiovascular risk, falling glomerular filtration rate, and finally terminal renal disease (ESRD). ${ }^{23}$

\section{Oxidative stress}

Oxidant species or reactive radicals are normally generated as by-products of normal cellular respiration/metabolism, and they play critical roles in 
cellular processes such as signalling, ageing, and degenerative disease. ${ }^{24}$ Anti-oxidant activity, which prevents potential harmful effects intermediate to excess oxidant accumulation, and oxidant species, which are considered to be essential signalling molecules for cellular physiology, coexist in perfect harmony in health. ${ }^{24}$ Acute oxidative stress and subsequent tissue injury can be caused by factors that cause an inequality in the oxidant species/anti-oxidant equivalence, which can manifest itself in the form of augmented oxidant species production or significantly reduced anti-oxidant activity. ${ }^{24} \mathrm{DN}$ has been associated with a variety of pathophysiological mechanisms, with augmented oxidant species being acknowledged as the single amalgamating upstream incident. ${ }^{25}$ In the pathophysiology of diabetic small blood vessel (microvascular) complications (retinopathy, nephropathy), oxidant species play a central and prominent role. $^{25}$

\section{Oxidative stress in kidney}

In capillaries, functional modification of the interaction between glomerular capillary endothelial cells and their glycocalyx layer, as well as the interaction between podocytes, occurs. ${ }^{25}$ All layers of the glomerular filtration apparatus are damaged by oxidant species. ${ }^{25}$ Afterwards, extracellular matrix dumping is observed, which is primarily characterised by an augmented expression of type IV collagen. ${ }^{25}$ Furthermore, the endothelial cell glycocalyx, which is primarily composed of proteoglycans and glycosaminoglycans with heparan sulphate and is recognised as a fundamental component of the glomerular filtration apparatus, is the most important target for reactive oxygen species. ${ }^{25}$ Exceedingly high levels of hydrogen peroxide result in the detachment of heparan sulfate from glycosaminoglycans and/or dilapidation of glycosaminoglycans, a secondary decrease in anionic charges, and an augmented ability of the kidneys to permeate macromolecules. $^{26}$

\section{NADPH oxidase}

The induction of matrix metalloproteinases and the inhibition of endogenous protease inhibitors are mediated by free radicals produced by oxygen and nitrogen, which has been predicted as a potential mechanism for glycocalyx dilapidation. ${ }^{27}$ The GBM, which is known to safeguard surface charge for the anionic heparan sulphate side chains appended to the core proteins agrin and perlecan, and the fine ground substance (ECM) topology, which is crucial to preserving the selectivity of filtering molecules, can be targets of surplus oxidant species fabrication. ${ }^{27}$ Hydroxyl radicals and other reactive species have been implicated in the breakdown of the polymeric chains of heparan sulphate and proteoglycan proteins, as well as protein dilapidation and cross-linking of type-IV collagen, both of which are directly involved in the preservation of the GBM's permeability discriminating properties. ${ }^{27}$ High levels of glucose and fatty acids in free form (FFA) in the blood have been found to be powerful activators of NADPH oxidase enzymes in diabetes research models. ${ }^{27}$ The RAAS has been noticed as one of the most imperative activators of NADPH oxidase and oxidant species ontogeny (origin and development). ${ }^{27,28}$ The diabetic kidney has been meticulously acknowledged for its powerful and standard role in the fabrication of vascular and renal reactive species. ${ }^{27,28}$ The physical link between NADPH-mediated superoxide anion and hydrogen peroxide synthesis and diabetic nephropathy pathogenesis is clear, as manifested by the augmented appearance of NADPH oxidase subunits in diabetic kidneys. ${ }^{27,28}$ The Nox 4 subtype has been recognized as the key basis of renal reactive species causing diabetic nephropathy, and has been the subject of extensive methodical investigation in recent years. ${ }^{28}$ In investigational replicates of diabetic nephropathy, inhibiting Nox 4 activity resulted in decreased oxidative stress and renal tissue injury. ${ }^{28}$ Nox 4 antisense oligonucleotides treatment reduced renal hypertrophy and mesangial cell expansion in diabetic animal replicates. ${ }^{28}$ In animal replicates of $\mathrm{DN}$, the renoprotective effects of knocking down Nox 4 were linked to a reduction in glomerular damage. ${ }^{28}$

\section{Nox in DN}

Diabetic nephropathy is characterized by oxidative damage, inflammation, and apoptosis. Reactive oxygen species (ROS), superoxide anion, and hydrogen peroxide $\left(\mathrm{H}_{2} \mathrm{O}_{2}\right)$ all play essential roles in various cellular alleyway networks. ${ }^{29}$ Amplified productions of ROS is thought to be a major downstream alleyway of end organ injury. ${ }^{29}$ Noxs, which include the isoforms Nox 1 to 5 , as well as Duox 1 and 2, cause DN. ${ }^{29}$

NOX-derived ROS acts as a mediator of kidney injury in a variety of hypertension and diabetes models. ${ }^{29}$ The kidney cortex expresses at least three different Nox isoforms, including Nox 4, Nox 2, and Nox 1. Nox 4 appears to be the most enriched with Nox. The Nox 4 isoform is over expressed in diabetic experimental models, and Nox 4 inhibition decreases glomerular expansion (hypertrophy), fibronectin fabrication, and proteinuria in diabetic models, implying a role for Nox 4-derived ROS in the pathogenesis of $\mathrm{DN}{ }^{29}$

\section{Nox inhibitors}

Studies on the role of the NADPH oxidases Nox 1 and Nox 4 in diabetic nephropathy: genetic deletion and pharmacological inhibition. Fluorofenidone (AKF-PD) is a new drug that has been shown to slow the progression of DN. In DN, Nox 4 deletion reduces oxidative stress and injury via PKC associated mechanisms. Crocin (paracetamol) improves kidney physiology by decreasing Nox 4, IL 18, and p53 expression levels in a DN experimental model. In an experimental model of DN, fenofibrate improves kidney physiology by improving NOX 4, IL 18, and p53 appearance. In an animal replicate of type 2 DN, probucol inhibited Nox 2 expression and 
reduced podocyte injury. In diabetic nephropathy, the Baoshenfang formula reduces podocyte injury by inhibiting the Nox-4/ROS/p38 alleyway. In an animal model, APX-115, a first-in-class pan-Nox inhibitor, protects against kidney injury. Resveratrol inhibits diabetic nephropathy and kidney interstitial fibrosis by regulating the AMPK/Nox 4/ROS alleyway. Plumbagin alleviates diabetic nephropathy by interfering with Nox 4 signaling alleyways. By quashing Nox-mediated oxidative stress and kidney inflammation, protocatechuic aldehyde quashes cisplatin-induced acute kidney injury. Melatonin's renoprotective effect is demonstrated in an animal model (Zucker diabetic fatty rats) due to its thwarting action on Nox. Delphinidin hampers Nox-1 and mitochondrial free radicals (superoxide radicals) in kidney mesangial cells, thwarting high glucose $(\mathrm{HG})$ provoked cell propagation and collagen production. ${ }^{75}$

Green tea (Camellia sinensis) reduces nephropathy in a diabetic animal model by down-regulating Nox 4 NADPH oxidase. Huidouba reduced podocyte injury in DN rats by down-regulating Nox 4 expression. Pitavastatin reduces albuminuria and kidney mesangial expansion in $\mathrm{db} / \mathrm{db}$ mice by down-regulating Nox 4. Pharmacological inhibition of albuminuria and matrix accumulation with the dual Nox 4/Nox 1 inhibitor GKT137831 reduces albuminuria and matrix accumulation. In streptozotocininduced diabetes rats, glycine reduces kidney oxidative stress by repressing Nox 4 expression. Naringin is used to manage diabetic nephropathy by inhibiting NADPH oxidase 4. The amplification in AMPK caused by cocoa has been revealed to be renoprotective in investigational diabetes mellitus by reducing Nox 4/TGF-1 alleyway. In a streptozotocin-induced diabetic rat model, valsartan reduces Nox 4 expression and prevents diabetic nephropathy. Zingerone reduces diabetic nephropathy by inhibiting the enzyme NADPH. Puerarin reduces diabetic kidney injury by controlling Nox 4 expression in podocytes.

Cudrania tricuspidata root extract reduces methylglyoxalinduced inflammation and oxidative stress via the PKCNox 4 Alleyway in vitro and in vivo. ${ }^{86}$ Huangqi decoction inhibits hyperglycemia-induced podocyte apoptosis by down-regulating the Nox 4/p53/Bax alleyway. Farrerol protects kidney mesangial cells from HG-induced injury via the ROS/Nox 4/ERK1/2 pathway. ${ }^{30-44}$

\section{Advantages}

It slows the development of $\mathrm{DN}$, reduces oxidative damage, improves renal function, reduces podocyte injury, prevents interstitial fibrosis, regulates AMPK, which inhibits ROS and TGF-beta, inhibits HG mediated activation of the ROS pathway, reduces albuminuria, decreases extracellular matrix deposition, decreases inflammation due to high glucose, protects mesangial cells, decreases collagen synthesis, reduces glomerular hypertrophy and, reduces fibronectin. ${ }^{22-44}$

\section{Disadvantages}

When tubular cells, cardiomyocytes, endothelial cells, and blood vessel (vascular) smooth muscle cells are injured, Nox 4 performs a cytoprotective and regulates metabolism. ${ }^{89}$ With the advent of pharmacological Nox 4 inhibitors in clinical trials, caution should be exercised in identifying potential side effects in patients who are predisposed to acute kidney injury and cardiovascular injury. ${ }^{45}$

\section{Precaution}

In clinical trials of pharmacological Nox 4 inhibitors, caution should be implemented in identifying potential side effects in patients with known ischemic heart disease, post myocardial infarction, and atherosclerosis. ${ }^{45}$

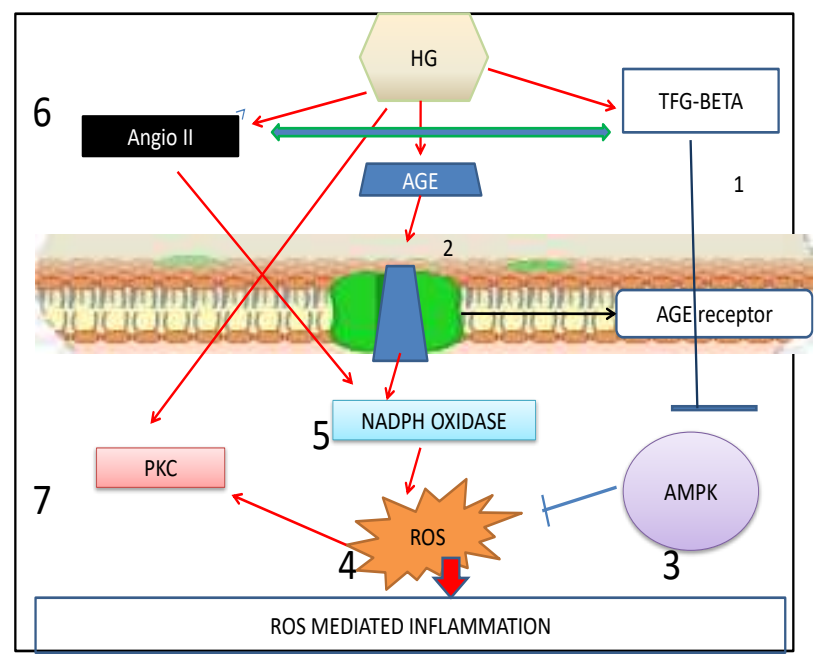

Figure 1: Mechanism of Nox activation, inhibitions and action of various activators and inhibitors used in management of $\mathrm{DN} .^{92-100}$

\section{DISCUSSION}

Many potential targets are reviewed for diabetic nephropathy. Nox inhibitors are tested from natural sources like green tea, plumbagin, Chinese formulas like Baoshenfang, Cudrania tricuspidata, Huangqi decoction and synthetic dual Nox inhibitors. Some of them activate and regulate AMPK. Some block the high glucose activated pathway, slowing the progression of DN. Overall, the benefits of Nox are: reducing oxidative damage, improving renal function, reducing podocyte injury, preventing interstitial fibrosis, regulating AMPK which inhibits ROS and TGF-beta, decreasing inflammation due to high glucose, protecting mesangial cells, decreasing collagen synthesis, reducing glomerular hypertrophy, and reducing fibronectin production. ${ }^{22-57}$ 


\section{Future challenge}

While targeting Nox renal impairment, off-target effects, especially cardiovascular effects, are one of the major hurdles, since DM is associated with co-morbid cardiovascular problems.

\section{CONCLUSION}

Nox inhibitors have the following benefits: quashing oxidative damage, mending renal function, reducing podocyte injury, thwarting interstitial fibrosis, regulating AMPK, which suppresses ROS and TGF-beta, decreasing inflammation caused by high glucose, shielding mesangial cells, inhibiting collagen synthesis, inhibiting glomerular hypertrophy, and inhibiting fibronectin production. Offtarget effects, especially on the cardiovascular system, are a substantial obstacle to Nox therapy in renal impairment, given that diabetes is associated with co-morbid cardiovascular issues.

\section{Recommendations}

Nox inhibitors can be game changers in the management of DN provided cardiovascular and other off-target effects are overpowered.

\section{ACKNOWLEDGEMENTS}

Authors would like to thank Sankalchand University, Gujarat, India.

Funding: No funding sources Conflict of interest: None declared

Ethical approval: Not required

\section{REFERENCES}

1. Piero MN, Nzaro GM, Njagi JM. Diabetes mellitus-a devastating metabolic disorder. Asian J Biomed Pharm Sci. 2015;5(40):1.

2. World Health Organization. Definition, diagnosis and classification of diabetes mellitus and its complications: report of a WHO consultation. Part 1, Diagnosis and classification of diabetes mellitus. 1999. Available at: https://apps.who.int/iris/handle /10665/66040. Accessed on 12 July 2021.

3. Wingard DL, Barrett-Connor E. Heart disease and diabetes. Diabetes in America. 1995;2(1):429-48.

4. Creager MA, Lüscher TF, Cosentino F, Beckman JA. Diabetes and vascular disease: pathophysiology, clinical consequences, and medical therapy: Part I. Circulation. 2003;108(12):1527-32.

5. Rudnisky CJ, Tennant MT, Johnson JA, Balko SU. Diabetes and eye disease in Alberta. Alberta diabetes atlas. 2011;141-62.

6. Koye DN, Magliano DJ, Nelson RG, Pavkov ME. The global epidemiology of diabetes and kidney disease. Adv Chronic Kidney Dis. 2018;25(2):121-32.
7. Jakobsen J. Peripheral nerves in early experimental diabetes. Diabetologia. 1978;14(2):113-9.

8. Bullard KM, Cowie CC, Lessem SE, Saydah SH, Menke A, Geiss LS, Orchard TJ, Rolka DB, Imperatore G. Prevalence of diagnosed diabetes in adults by diabetes type-United States, 2016. Morb Mort Wkly Rep. 2018;67(12):359.

9. Meneilly GS, Tessier D. Diabetes in elderly adults. J Gerontol Series. 2001;56(1):5-13.

10. Petersen KF, Shulman GI. Etiology of insulin resistance. Am J Med. 2006;119(5):10-6.

11. Wallace TM, Matthews DR. The assessment of insulin resistance in man. Diabet Med. 2002;19(7):527-34.

12. Chan C, Harper ME. Uncoupling proteins: role in insulin resistance and insulin insufficiency. Curr Diabet Rev. 2006;2(3):271-83.

13. Khan MA, Hashim MJ, King JK, Govender RD, Mustafa H, Al Kaabi J. Epidemiology of type 2 diabetes-global burden of disease and forecasted trends. J Epidemiol Global Health. 2020;10(1):107.

14. Kaiser AB, Zhang N, Van Der Pluijm W. Global prevalence of type 2 diabetes over the next ten years (2018-2028). Diabetes. 2018;67(1).

15. Cooke DW, Plotnick L. Type 1 diabetes mellitus in pediatrics. Pediatr Rev. 2008;29(11):374-84.

16. Katsarou A, Gudbjörnsdottir S, Rawshani A, Dabelea D, Bonifacio E, Anderson BJ, Jacobsen LM, Schatz DA, Lernmark A. Type 1 diabetes mellitus. Nature Rev Dis Primers. 2017;3(1):1-7.

17. Steele C, Hagopian WA, Gitelman S, Masharani U, Cavaghan M, Rother KI, et al. Insulin secretion in type 1 diabetes. Diabetes. 2004;53(2):426-33.

18. Dall TM, Zhang Y, Chen YJ, Quick WW, Yang WG, Fogli J. The economic burden of diabetes. Health Affairs. 2010;29(2):297-303.

19. Bommer C, Heesemann E, Sagalova V, ManneGoehler J, Atun R, Bärnighausen T, Vollmer S. The global economic burden of diabetes in adults aged 20 79 years: a cost-of-illness study. Lancet Diabet Endocrinol. 2017;5(6):423-30.

20. Valdmanis V, Smith DW, Page MR. Productivity and economic burden associated with diabetes. Am $\mathbf{J}$ Public Health. 2001;91(1):129.

21. Lin X, Xu Y, Pan X, Xu J, Ding Y, Sun X, et al. Global, regional, and national burden and trend of diabetes in 195 countries and territories: an analysis from 1990 to 2025. Scientific Rep. 2020;10(1):1-1.

22. World Health Organization. Diabetes. Available at: https://www.who.int/health-topics/diabetes\#tab=tab_ 1. Accessed on 24 June 2021.

23. Martínez-Castelao A, Navarro-González JF, Górriz JL, De Alvaro F. The concept and the epidemiology of diabetic nephropathy have changed in recent years. $\mathbf{J}$ Clin Med. 2015;4(6):1207-16.

24. Quijano C, Trujillo M, Castro L, Trostchansky A. Interplay between oxidant species and energy metabolism. Redox Biol. 2016;8:28-42.

25. Ratliff BB, Abdulmahdi W, Pawar R, Wolin MS. Oxidant mechanisms in renal injury and disease. Antioxidants \& redox signaling. 2016;25(3):119-46. 
26. Maestroni S, Zerbini G. Glomerular endothelial cells versus podocytes as the cellular target in diabetic nephropathy. Acta diabetologica. 2018;55(11):110511 .

27. Singh A, Ramnath RD, Foster RR, Wylie EC, Fridén V, Dasgupta I, Haraldsson B, Welsh GI, Mathieson PW, Satchell SC. Reactive oxygen species modulate the barrier function of the human glomerular endothelial glycocalyx. PloS one. 2013;8(2):e55852.

28. Sedeek M, Nasrallah R, Touyz RM, Hébert RL. NADPH oxidases, reactive oxygen species, and the kidney: friend and foe. J Am Soc Nephrol. 2013;24(10):1512-8.

29. Lee SR, An EJ, Kim J, Bae YS. Function of NADPH oxidases in diabetic nephropathy and development of nox inhibitors. Biomol Therap. 2020;28(1):25.

30. Augsburger F, Filippova A, Rasti D, Seredenina T, Lam M, Maghzal G, et al. Pharmacological characterization of the seven human NOX isoforms and their inhibitors. Redox Biol. 2019;26:101272.

31. Elbatreek MH, Mucke H, Schmidt HH. NOX inhibitors: from bench to naxibs to bedside. Handb Exp Pharmacol. 2021;264:145-68.

32. Rastogi R, Geng X, Li F, Ding Y. NOX activation by subunit interaction and underlying mechanisms in disease. Front Cellular Neurosci. 2017;10:301.

33. Zielonka J, Zielonka M, Cheng G, Hardy M, Kalyanaraman B. High-throughput screening of NOX inhibitors. NADPH Oxidases. Humana, New York, NY. 2019;429-46.

34. Laddha AP, Kulkarni YA. NADPH oxidase: A membrane-bound enzyme and its inhibitors in diabetic complications. Eur J Pharmacol. 2020;881:173206.

35. Reis J, Massari M, Marchese S, Ceccon M, Aalbers FS, Corana F, et al. A closer look into NADPH oxidase inhibitors: Validation and insight into their mechanism of action. Redox Biol. 2020;32:101466.

36. Urner S, Ho F, Jha JC, Ziegler D, Jandeleit-Dahm K. NADPH oxidase inhibition: preclinical and clinical studies in diabetic complications. Antioxidants \& redox signaling. 2020;33(6):415-34.

37. Zhang J, Yang S, Li H, Chen F, Shi J. Naringin ameliorates diabetic nephropathy by inhibiting NADPH oxidase 4. European journal of pharmacology. 2017;804:1-6.

38. Papadimitriou A, Peixoto EB, Silva KC, de Faria JM, de Faria JB. Increase in AMPK brought about by cocoa is renoprotective in experimental diabetes mellitus by reducing NOX4/TGF $\beta-1$ signaling. J Nutritional Biochem. 2014;25(7):773-84.

39. Zhou G, Cheung AK, Liu X, Huang Y. Valsartan slows the progression of diabetic nephropathy in $\mathrm{db} / \mathrm{db}$ mice via a reduction in podocyte injury, and renal oxidative stress and inflammation. Clin Sci. 2014;126(10):707-20.

40. Cui Y, Shi Y, Bao Y, Wang S, Hua Q, Liu Y. Zingerone attenuates diabetic nephropathy through inhibition of nicotinamide adenine dinucleotide phosphate oxidase 4. Biomed Pharmacotherap. 2018;99:422-30.
41. Li X, Cai W, Lee K, Liu B, Deng Y, Chen Y, Zhang $\mathrm{X}$, He JC, Zhong Y. Puerarin attenuates diabetic kidney injury through the suppression of NOX4 expression in podocytes. Scientific Rep. 2017;7(1):11.

42. Kim D, Cheon J, Yoon H, Jun HS. Cudrania tricuspidata Root Extract Prevents MethylglyoxalInduced Inflammation and Oxidative Stress via Regulation of the PKC-NOX4 Pathway in Human Kidney Cells. Oxidative medicine and cellular longevity. 2021.

43. Li Z, Deng W, Cao A, Zang Y, Wang Y, Wang H, Wang L, Peng W. Huangqi decoction inhibits hyperglycemia-induced podocyte apoptosis by downregulated Nox4/p53/Bax signaling in vitro and in vivo. Am J Transl Res. 2019;11(5):3195.

44. Chen Z, Gao H, Wang L, Ma X, Tian L, Zhao W, et al. Farrerol alleviates high glucose-induced renal mesangial cell injury through the ROS/Nox4/ERK1/2 pathway. Chemico-biological interactions. 2020;316:108921.

45. Rajaram RD, Dissard R, Jaquet V, de Seigneux S. Potential benefits and harms of NADPH oxidase type 4 in the kidneys and cardiovascular system. Nephrology Dialysis Transplantation. 2019;34(4):567-76.

46. Soni NO. Drugs for Diabetic Nephropathy-full review. World J Pharm Pharmaceutical Sci. 2017;6(7):19582022.

47. Soni NO. Embryonic life of HDACs inhibitor-in diabetic nephropathy. World J Pharm Pharmaceutical Sci. 2017;6:345-59.

48. Soni NO. TRPC 6 as a Molecular Target in Diabetic Nephropathy. Int $\mathbf{J}$ Life Sci Scienti Res. 2017;3(5):1311-4.

49. Soni NO. Resveratrol nanoparticle's formulation-in diabetic nephropathy. World J Pharm Pharmaceutical Sci. 2017;368-86.

50. Soni NO. Biodegradable Nanoparticles for Delivering Drugs and Silencing Multiple Genes or Gene activation in Diabetic Nephropathy. Int J Life Sci Scienti Res. 2017;3(5):1329-38.

51. Soni NO. Targeting DNA methyl transferase 1 [dnmt1]-in diabetic nephropathy. World J Pharm Pharmaceutical Sci. 2017;6(9):333-38.

52. Soni NO. Monaschin and ankaflavin-in diabetic nephropathy? World J Pharm Pharmaceutical Sci. 2017;6(9):327-32.

53. Soni NO, Pawar SV, Kale S, Mane UA, Bhosale PU, Phandhare K, Bhosale PR, Patil SA. Peroxisome proliferator-activated receptors and thiazolidinediones in diabetic nephropathy. Int J Basic Clin Pharmacol. 2019;8(10):2344.

54. Soni NO. NADPH oxidase is novel drug in diabetic nephropathy. World J Pharm Pharmaceutical Sci. 2017;6:765-5.

55. Soni NO. TGF-'Inhibitors Are Novel Drugs-In Diabetic Nephropathy a Review. World J Pharm Pharmaceutical Sci. 2017;622-45. 
56. Soni NO. Recombinant complement inhibitor CD59in diabetic nephropathy. World $\mathrm{J}$ Pharm Pharmaceutical Sci. 2017;6(7):2056-60.

57. Soni NO. Fructokinase inhibitors-in diabetic nephropathy. World J Pharm Pharmaceutical Sci. 2017;6(7):339-44.
Cite this article as: Soni NO. A brief study of Nox 4 inhibitors in diabetic nephropathy. Int J Basic Clin Pharmacol 2021;10:1311-6. 